Cyber-205 machines with a massive shared memory. At the other end of the spectrum, fine-grained parallel machines such as the Connection Machine use many simple processors which communicate with each other via a complicated network of interconnections.

The optimal algorithm for solving the gravitational $n$-body problem clearly depends on the architecture of the machine. Existing programs such as Aarseth's famous $n$-body code can easily be adapted for vector machines and will be used extensively at the supercomputer centres. Algorithms for very fine-grained computers (which use many simple processors) are likely to be quite different. Several ingenious possibilities were described at the meeting (Joshua Barnes, Institute for Advanced Study, Princeton).

Very few cosmologists and astrophysicists have thought seriously about building their own special-purpose supercomputer, yet it is feasible to acquire the necessary skills (Gerald Sussman, MIT). Sussman also argued that it is now possible to build one-off machines to study the stellar dynamical evolution of, say, an entire globular star cluster containing one million stars.

But even the globular cluster problem is not entirely stellar dynamical. At some point during evolution, the finite sizes of stars may become important, as may mass-loss and other astrophysical effects. In the case of cosmological simulations, our lack of knowledge of gas dynamics and star formation impose severe limitations on our understanding. Supercomputers will undoubtedly make a major impact in cosmology and astrophysics, but in many cases they will solve only idealized problems. Theorists will still have plenty to do in applying the results to real stellar systems.

George Efstathiou is at the Institute of Astronomy, University of Cambridge, Madingley Road, Cambridge CB3 OHA, UK.

\title{
Nitrogen fixation
}

\section{A role for vanadium at last}

\section{from R. Cammack}

FIFTY years after Bortels reported ${ }^{1}$ that vanadium could substitute for molybdenum as a trace element essential for nitrogen fixation in the soil bacterium Azotobacter vinelandii, Robson et al., on page 388 of this issue ${ }^{2}$, report conclusive evidence for an alternative vanadiumcontaining nitrogenase from $A$. chroococcum, a result made possible by recent developments in the genetics of nitrogenfixing bacteria. Robson et al. produced a mutant strain in which the structural genes for the normal molybdenum-containing nitrogenase are deleted and demonstrate that the alternative nitrogenase uniquely requires vanadium for its synthesis, and in stoichiometric amounts.

The complex, nitrogen-fixing enzyme nitrogenase has two types of protein component, both containing iron-sulphur clusters. One of these components, the iron-molybdenum protein, also contains a cluster of unknown structure, the iron-molybdenum cofactor ( $\mathrm{Fe}-\mathrm{Mo}-\mathrm{Co})$ : there is compelling evidence that this is the site at which dinitrogen is reduced to ammonia. The function of the other component is to 'pump' electrons, using energy from ATP, into the iron-molybdenum protein. The vanadium-containing nitrogenase appears to have a very similar arrangement, a structure containing vanadium presumably substituting for the $\mathrm{Fe}-\mathrm{Mo}-\mathrm{Co}$, although the proteins involved are the products of different genes.

Chemically, the substitution of vanadium for molybdenum makes good sense. The two elements are capable of similar oxidation-reduction reactions at low redox potentials. Model compounds for the nitrogenase reaction that can catalyse the reduction of dinitrogen to ammonia have been synthesized, most containing molybdenum, although complexes of vanadium are also effective ${ }^{3}$.

Ironically, although nitrogenase was partially purified from vanadium-grown $A$. vinelandii about 15 years ago and several of its catalytic properties, such as decreased sensitivity to cobalt inhibition, correctly described, the significance of vanadium in this system was not recognized. This was partly because of the extreme difficulty of obtaining growth media that were sufficiently molybdenum-free, which meant that all nitrogenase preparations contained a certain amount of molybdenum. It must have seemed unreasonable at the time to assume that there existed a second nitrogenase system with almost identical protein structure to the molybdenum nitrogenase system, which rapidly decomposes during purification and has very little activity in the conventional acetylene-reduction assay. Instead it was concluded that vanadium had a 'sparing' effect, stabilizing the molybdenum nitrogenase when molybdenum was in short supply. The idea that all nitrogenases contain molybdenum became firmly established and molybdenum was routinely added to all growth media for nitrogen-fixing organisms. Because the synthesis of the vanadium nitrogenase is repressed even by traces of molybdenum, its presence was not detected. A recent survey of the literature showed that Bortels' 1930 paper describing a requirement for molybdenum in nitrogen fixation has been cited 55 times, while his 1936 paper $^{4}$ describing the alternative use of vanadium was cited 4 times.

The more recent discoveries stem from the work of Bishop ${ }^{5}$ who, in the face of much initial scepticism, demonstrated the presence of a second nitrogenase system in $A$. vinelandii grown in molybdenumdeficient conditions. He obtained a nif mutant which could not express the conventional molybdenum nitrogenase, but which could still grow by fixing nitrogen in the absence of molybdenum. He showed that these 'pseudo-revertants' were tungstate resistant and expressed a different nitrogenase whose activity (as measured by hydrogen production) was less sensitive to inhibition by acetylene, carbon monoxide or tungstate. It appears that it was the vanadium present as a contaminant in tungstate that allowed Robson et $a l{ }^{2}$ to discover the vanadium enzyme.

Azotobacter synthesizes the molybdenum nitrogenase in preference to the vanadium nitrogenase when molybdenum is available presumably because the molybdenum enzyme is more stable, and possibly catalyses less of the wasteful side-reaction in which hydrogen is produced. Although molybdenum is difficult to remove from laboratory media, it may become a limiting factor in some soils, and hence the capacity to make the alternative nitrogenase confers a selective advantage. A question of great interest is whether other nitrogen-fixing organisms, and in particular the economically important rhizobia, are capable of making the vanadium nitrogenase.

Macara ${ }^{6}$ has described vanadium as "an element in search of a [biochemical] role". It is known that vanadium in extremely small amounts is a nutritional requirement for many types of organism, possibly including higher animals ${ }^{7}$. The sea-squirt Ascidia nigra contains high concentrations of V(III) ions in specialized blood cells. Vanadium is also accumulated by the toadstool Amanita muscaria ${ }^{8}$ and a vanadium-containing bromoperoxidase has recently been isolated from a seaweed, Ascophyllum nodosum ${ }^{9}$. With the establishment of vanadium in nitrogenase, its role is beginning to emerge.

1. Bortels. H. Zentbl. Bakt. ParasitKde Abt. II 95, 193 (1936) 2. Robson, R.L.et al. Nature 322, 388 (1986).

3. Nikonova, L.A., Isaeva, S.A., Pershikova, N.I. \& Shiłov, A.E. J. molec. Catal. 1. 367 (1975)

4. Bortels H. Arch. Mikrobiol. 1, 333 (1930).

5. Bishop, P. Trends biochem. Sci. 11, 225 (1986)

6. Macara, I. G. Trends biochem. Sci. 5, $92(1980)$

7. Nechay, B.R. Fedn Proc. 45.123 (1986).

8. Gillard, R.D. \& Lancashire. R.J. Phytochemistry 23, 179 (1983)

9. De Boer, E., Van Kooyk, Y., 'Tromp, M.G.M., Plat, H. \& Wever, R. Biochim. biophys. Acta 869, 48 (1986).

R. Cammack is a Reader in the Department of Biochemistry, King's College London, Campden Hill Road, London W87AH, UK. 